九州大学学術情報リポジトリ

Kyushu University Institutional Repository

\title{
AN APPLICATION OF A COMBINATORIAL THEOREM TO A SEPARATION PROBLEM II
}

Maruyama, Fumio

Department of Economics, Miyazaki Sangyo-Keiei University

https://doi.org/10.5109/13497

出版情報: Bulletin of informatics and cybernetics. 32 (2)，pp.123-138，2000-12. Research Association of Statistical Sciences

バージョン :

権利関係 : 


\title{
AN APPLICATION OF A COMBINATORIAL THEOREM TO A SEPARATION PROBLEM II
}

By

\author{
Fumio MaruYama*
}

\begin{abstract}
On the space of real-valued functions of several variables, we defined a partially ordered set of functionals $(\Sigma, \leqslant)$ and proved a separation theorem (Maruyama,2000). In this paper, an ordering theorem is proved: for an arbitrary order refinement $(\Sigma, \preccurlyeq)$, we construct a function $F$ such that $\tau \prec \tau^{\prime}$ implies $\tau(F)<\tau^{\prime}(F)$.
\end{abstract}

\section{Introduction}

On the space of real-valued functions of several variables a sequential optimal choices of variables determines a functional. Typical examples of such functionals are the optimal plays of two players in finite zero-sum dynamic games of perfect information between minimizer and maximizer, which assign game values to payoff functions. In such a case each choice of variable depends on the previous choices of variables. Clearly the set of previous choices of variables is linearly ordered by set inclusion. In this sense we can say dependency of variables is linearly ordered.

By considering optimizations under nonlinearly ordered dependencies of variables, we generalized such functionals and defined a set of functionals $\Sigma$ in Maruyama (2000). In the study of the natural pointwise order on $\Sigma$, utilizing the phenomenon that any sufficiently large structure contains a well regulated substructure, we proved a separation theorem. Precisely, by the use of the theorem of Hales-Jewett (cf. Graham, Rothschild and Spencer,1990), we proved that for functionals $\tau, \tau_{1}, \tau_{2}, \ldots, \tau_{t} \in \Sigma$, if there exists a function $F_{i}$ such that $\tau\left(F_{i}\right)<\tau_{i}\left(F_{i}\right)$ (resp. $\tau\left(F_{i}\right)>\tau_{i}\left(F_{i}\right)$ ) for each $i \in\{1,2, \ldots, t\}$, then there exists a function $F$ such that $\tau(F)<\tau_{i}(F)$ (resp. $\tau(F)>\tau_{i}(F)$ ) for all $i \in\{1,2, \ldots, t\}$ when the domain of each variable has sufficiently many elements.

In the linear case the analysis is not difficult. In fact, much more stronger result than this separation theorem was obtained in Hisano and Maruyama (1989). It was proved that if a subset $S$ of $\Sigma$ consists of functionals of linearly ordered dependency of variables, then any total ordering on $S$ can be assigned by a function as long as it is consistent with the natural partial order. More precisely, if $\tau_{1}, \tau_{2}, \ldots, \tau_{t}$ are functionals defined by sequential optimal choices of variables and if there exists a function $F_{i j}$ such that $\tau_{i}\left(F_{i j}\right)<\tau_{j}\left(F_{i j}\right)$ for all $i, j \in\{1,2, \ldots, t\}$ with $i<j$, then there exists a function $F$ such that $\tau_{1}(F)<\tau_{2}(F)<\cdots<\tau_{t}(F)$.

In this paper, as a strengthening of the separation theorem, we generalize this result to nonlinear case and clarify the order structure on $\Sigma$.

From the point of view of logic, the set of functionals $\Sigma$ corresponds to a set of nonlinear quantifier prefixes (cf. Krynicki and Mostowski,1995) and their dual quantifier

\footnotetext{
* Department of Economics, Miyazaki Sangyo-Kejei University, Miyakonojo 885-0035, Japan
} 
prefixes, functionals of linear case correspond to linear quantifier prefixes and the order relations between functionals correspond to implication relations between quantifier prefixes. Hence, by the result in this paper, it maybe observed that as in the case of linear quantifier prefixes there exists no nontrivial implication relation among nonlinear quantifier prefixes and their duals.

\section{Notation and statement of the main result}

We denote the cardinality of a set $U$ by $\sharp U$. For a set $U$ and a cardinal $k$ we write $\left(\begin{array}{c}U \\ k\end{array}\right)=\{V \subset U \mid \sharp V=k\}$. Denote by ${ }^{U} V$ the set of all mappings from a set $U$ into a set $V$. We note that ${ }^{\phi} V=\{\phi\}$. Denote by $\mathfrak{D}(f)$ and $\mathfrak{R}(f)$ the domain and the range of a mapping $f$ respectively.

For integers $k$ and $l,[k, l]$ denotes the set of integers larger than $k-1$ and less than $l+1$. We denote by $\boldsymbol{N}, \boldsymbol{R}$, respectively, the set of all positive integers, and the set of all real numbers. We write $\overrightarrow{\boldsymbol{R}}=\boldsymbol{R} \cup\{-\infty, \infty\}$.

Let $n \in N$ be fixed. For each $k \in[1, n]$ let $Z^{k}$ be a set which contains at least two elements. We set

$$
\begin{aligned}
& \Pi_{k}=\left\{\eta \in^{[1, k]}[1, n] \mid \sharp \eta([1, k])=k\right\}(1 \leqslant k \leqslant n), \Pi=\Pi_{n}, \Pi_{0}=\{\phi\}, \\
& \Gamma(\pi, l)=\left\{\gamma=\left(\gamma_{1}, \ldots, \gamma_{l}\right) \in\left(\bigcup_{k=0}^{n-l} \Pi_{k}\right)^{l} \mid \bigcup_{k=1}^{l} \Re\left(\gamma_{k}\right) \subset \pi([l+1, n])\right\} \\
& \Gamma(\pi, 0)=\{\phi\}, \\
& \Phi(l, \eta)={ }^{\left(Z^{(1)} \times \cdots \times Z^{\eta(k)}\right)} Z^{l} \quad\left(k, l \in[1, n], \eta \in \Pi_{k}\right), \\
& \Phi(l, \phi)=Z^{l} \quad(1 \leqslant l \leqslant n) .
\end{aligned}
$$

For $F \in^{\left(Z^{1} \times \cdots \times Z^{n}\right)} R, \pi \in \Pi, l \in[1, n], \gamma \in \Gamma(\pi, l)$ define

$$
\pi * F: Z^{\pi(1)} \times \cdots \times Z^{\pi(n)} \longrightarrow \boldsymbol{R}
$$

and

$$
\gamma * \pi * F: \Phi\left(\pi(1), \gamma_{1}\right) \times \cdots \times \Phi\left(\pi(l), \gamma_{l}\right) \times Z^{\pi(l+1)} \times \cdots \times Z^{\pi(\pi)} \longrightarrow R
$$

by

$$
(\pi * F)\left(z^{\pi(1)}, \ldots, z^{\pi(n)}\right)=F\left(z^{1}, \ldots, z^{n}\right)
$$

and

$$
\begin{aligned}
& (\gamma * \pi * F)\left(\varphi^{1}, \ldots, \varphi^{l}, z^{\pi(l+1)}, \ldots, z^{\pi(n)}\right) \\
& =(\pi * F)\left(\varphi^{1}\left(z^{\gamma_{1}(1)}, \ldots, z^{\gamma_{1}\left(h_{1}\right)}\right), \ldots, \varphi^{l}\left(z^{\gamma_{l}(1)}, \ldots, z^{\gamma_{l}\left(h_{1}\right)}\right), z^{\pi(l+1)}, \ldots, z^{\pi(n)}\right)
\end{aligned}
$$

respectively for all $\varphi^{1} \in \Phi\left(\pi(1), \gamma_{1}\right), \ldots, \varphi^{l} \in \Phi\left(\pi(l), \gamma_{l}\right)$ and $z^{\pi(l+1)} \in Z^{\pi(l+1)}, \ldots$, $z^{\pi(n)} \in Z^{\pi(n)}$ where $\mathfrak{D}\left(\gamma_{k}\right)=\left[1, h_{k}\right](1 \leqslant k \leqslant l)$. We set $\phi * \pi * F=\pi * F$. 
For $l \in[0, n], \pi \in \Pi, \gamma \in \Gamma(\pi, l)$ define

$$
\sigma_{+}(l, \pi, \gamma), \sigma_{-}(l, \pi, \gamma):{ }^{\left(Z^{1} \times \cdots \times Z^{n}\right)} \boldsymbol{R} \longrightarrow \overline{\boldsymbol{R}}
$$

by

$$
\begin{aligned}
& \sigma_{+}(l, \pi, \gamma)(F)=\sup _{\varphi^{1} \in \Phi\left(\pi(1), \gamma_{1}\right)} \cdots \sup _{\varphi^{1} \in \Phi\left(\pi(l), \gamma_{l}\right)} \inf _{z^{\pi(l+1)} \in Z^{\pi(I+1)}} \cdots \inf _{z^{\pi(n)} \in Z^{*(n)}} \\
& (\gamma * \pi * F)\left(\varphi^{1}, \ldots, \varphi^{l}, z^{\pi(l+1)}, \ldots, z^{\pi(n)}\right), \\
& \sigma_{-}(l, \pi, \gamma)(F)=\inf _{\varphi^{1} \in \Phi\left(\pi(1), \gamma_{1}\right)} \cdots \inf _{\varphi^{\prime} \in \Phi\left(\pi(l), \gamma_{l}\right)} \sup _{z^{\pi(l+1)} \in Z^{*(l+1)}} \cdots \sup _{z^{\pi(n)} \in Z^{\pi(n)}} \\
& (\gamma * \pi * F)\left(\varphi^{1}, \ldots, \varphi^{l}, z^{\pi(l+1)}, \ldots, z^{\pi(n)}\right) .
\end{aligned}
$$

We set

$$
\begin{aligned}
& \Sigma_{+}=\left\{\sigma_{+}(l, \pi, \gamma) \epsilon^{\left\{Z^{l} \times \cdots \times Z^{n}\right)} \boldsymbol{R} \bar{R} \mid 0 \leqslant l \leqslant n, \pi \in \Pi, \gamma \in \Gamma(\pi, l)\right\}, \\
& \Sigma_{-}=\left\{\sigma_{-}(l, \pi, \gamma) \epsilon^{\left(z^{1} \times \cdots \times z^{n}\right)} \boldsymbol{R} \bar{R} \mid 0 \leqslant l \leqslant n, \pi \in \Pi, \gamma \in \Gamma(\pi, l)\right\}, \\
& \Sigma=\Sigma_{+} \cup \Sigma_{-} .
\end{aligned}
$$

For $\nu=\sigma_{+}(l, \pi, \gamma), \mu=\sigma_{-}(l, \pi, \gamma) \in \Sigma$ we set

$$
\begin{aligned}
& \bar{\nu}=\underline{\mu}=\pi([1, l]), \\
& \underline{\nu}=\bar{\mu}=\pi([l+1, n]), \\
& \nu_{+}=\mu_{-}=\bigcup_{k=1}^{l}\left\{(u, \pi(k)) \in[1, n]^{2} \mid u \in \Re\left(\gamma_{k}\right)\right\}, \\
& \nu_{-}=\mu_{+}=\bigcup_{k=1}^{l}\left\{(\pi(k), u) \in[1, n]^{2} \mid u \in \pi([l+1, n]) \backslash \Re\left(\gamma_{k}\right)\right\} .
\end{aligned}
$$

For $\tau, \tau^{\prime} \in \Sigma$ and $p \in[1,[n / 2]]$ (where $[n / 2]$ denotes the largest integer not exceeding $n / 2$ ) define

$$
\begin{aligned}
C\left(\tau, \tau^{\prime}, p\right)=\left\{\eta \in \Pi_{2 p} \mid(\eta(p+k), \eta(k)) \in \tau_{+}^{\prime}(1 \leqslant k \leqslant p),\right. \\
\left.(\eta(k), \eta(p+k+1)) \in \tau_{-}(1 \leqslant k \leqslant p-1),(\eta(p), \eta(p+1)) \in \tau_{-}\right\} .
\end{aligned}
$$

We set $C\left(\tau, \tau^{\prime}, 0\right)=\underline{\tau} \cap \overline{\tau^{\prime}}$.

We define the order $\leqslant$ on $\Sigma$ by, for $\tau, \tau^{\prime} \in \Sigma$,

$\tau \leqslant \tau^{\prime} \quad$ if and only if $\quad \tau(F) \leqslant \tau^{\prime}(F)$ for all $F: Z^{1} \times Z^{2} \times \cdots \times Z^{n} \rightarrow \boldsymbol{R}$.

Then, when the cardinals of $Z^{1}, Z^{2}, \ldots, Z^{n}$ are large, every order refinement of the ordered set $(\Sigma, \leqslant)$ can be refined by a function in $\left(Z^{1} \times Z^{2} \times \cdots \times Z^{n}\right) R$.

This follows from the following ordering theorem.

Theorem 2.1 (ordering theorem). Assume that $Z^{1}, Z^{2}, \ldots, Z^{n}$ have sufficiently many elements. Then for any $\tau_{1}, \tau_{2}, \ldots, \tau_{t} \in \Sigma$ such that $\tau_{j} \tau_{i}(1 \leqslant i<j \leqslant t)$, there exists $F: Z^{1} \times Z^{2} \times \cdots \times Z^{n} \longrightarrow \boldsymbol{R}$ such that $\tau_{1}(F)<\tau_{2}(F)<\cdots<\tau_{t}(F)$. 


\section{Proof of the theorem} lemmas.

In this section we prove the ordering theorem. We begin with some definitions and

Throughout the following definitions and lemmas $\tau_{1}, \tau_{2}, \ldots, \tau_{t}$ will be arbitrary but fixed elements of $\Sigma$ satisfying $\tau_{i} \tau_{j}(1 \leqslant j<i \leqslant t)$.

For $s \in[1, t]$ let $l_{s}, \pi_{s}, \gamma^{s}$ be such that $r_{s}=\sigma_{+}\left(l_{s}, \pi_{s}, \gamma^{s}\right)$ for $\tau_{s} \in \Sigma_{+}, \tau_{s}=$ $\sigma_{-}\left(l_{s}, \pi_{s}, \gamma^{s}\right)$ for $\tau_{s} \notin \Sigma_{+}$. For $u, v \in[1, t]$ with $v<u$ define

$$
p(u, v)=\min \left\{k \in[0,[n / 2]] \mid C\left(\tau_{v}, \tau_{u}, k\right) \neq \phi\right\}
$$

and fix $\rho_{u, u} \in C\left(\tau_{v}, \tau_{u}, p(u, v)\right)$. Set

$$
\begin{aligned}
& V(v, u) \\
& =\left(\pi_{v}\left(\left[l_{v}+1, n\right]\right) \times \bigcup_{s=2}^{t}(\{s\} \times[1, s-1])\right) \backslash\left(\rho_{u, v}([p(u, v)+1,2 p(u, v)]) \times\{(u, v)\}\right), \\
& V(u, v)=\pi_{u}\left(\left[l_{\mathfrak{u}}+1, n\right]\right) \times\left[\bigcup_{s \in[2, t] \backslash\{u\}}(\{s\} \times[1, s-1]) \cup(\{u\} \times[1, v])\right], \\
& V(u, 0)=\pi_{\mathfrak{u}}\left(\left[l_{u}+1, n\right]\right) \times \bigcup_{s \in[2, t] \backslash\{u\}}(\{s\} \times[1, s-1]) .
\end{aligned}
$$

If $p(u, v) \geqslant 1$ and $1 \leqslant k \leqslant p(u, v)$ we set

$$
\begin{aligned}
& U(u, v, k)=\left\{\begin{array}{l}
\mathfrak{N}\left(\gamma_{\pi_{u}^{-1} \circ \rho_{u, v}(k)}^{u}\right)\left(\tau_{u} \in \Sigma_{+}\right), \\
\Re\left(\gamma_{\pi_{u}^{-1} \circ \rho_{u, v}(p(u, v)+k)}^{u}\right)\left(\tau_{u} \notin \Sigma_{+}\right),
\end{array}\right. \\
& U(v, u, k)=\left\{\begin{array}{l}
\mathfrak{M}\left(\gamma_{\pi_{v}^{-1} \circ \rho_{u, v}(k)}^{v}\right)\left(\tau_{v} \in \Sigma_{+}\right), \\
\mathfrak{M}\left(\gamma_{\pi_{v}^{-1} \circ \rho_{u, v}(p(u, v)+k)}^{v}\right)\left(\tau_{v} \notin \Sigma_{+}\right) .
\end{array}\right.
\end{aligned}
$$

Definition 3.1. For $1 \leqslant v<u \leqslant t, 1 \leqslant k \leqslant n$ define $X_{u, v}^{k}=X_{u, v}^{k}\left(\tau_{1}, \ldots, \tau_{t}\right)$ and $Y_{u, v}^{k}=Y_{u, v}^{k}\left(\tau_{1}, \ldots, \tau_{t}\right)$ as follows.

If $p(u, v)=0$, then $X_{u, v}^{k}=Y_{u, v}^{k}=\{0\}(1 \leqslant k \leqslant n)$.

If $p(u, v) \geqslant 1$, then for $k \notin \rho_{u, v}([1, p(u, v)]) X_{u, v}^{k}=\{0\}$ and for $k \notin \rho_{u, v}([p(u, v)+$ $1,2 p(u, v)]) Y_{u, v}^{k}=\{0\}$. Furthermore, for $k \in[1, p(u, v)]$ we write $X(u, v, k)=X_{u, v}^{\rho_{u, v}(k)}$ and $Y(u, v, k)=Y_{u, v}^{\rho_{u, v}}(p(u, v)+k)$.

For $\tau_{u} \in \Sigma_{+}$,

if $\tau_{v} \notin \Sigma_{+}$, then $X(u, v, k)=Y(u, v, k)=\{0,1\}$,

if $\tau_{v} \in \Sigma_{+}$, then $X(u, v, k)=Y(u, v, k)=[1, t]$.

For $\tau_{u} \notin \Sigma_{+}$, we define $X(u, v, k), Y(u, v, k)$ inductively as follows.

If $p(u, v)=1$, then $X(u, v, 1)=[1, \sharp W(u, v, 1)], Y(u, v, 1)=\left[1, N_{u, v}\right]$ 
where

$$
N_{u, v}=t \cdot \prod_{k \in U(u, v, 1)} \prod_{v+1 \leqslant s \leqslant u-1} \sharp X_{u, s}^{k}
$$

and

$$
W(u, v, 1)=\left(\begin{array}{c}
{\left[1, N_{u, v}\right]} \\
\prod_{k \in U(u, v, 1)} \prod_{v+1 \leqslant s \leqslant u-1} \sharp X_{u, s}^{k}
\end{array}\right) .
$$

We set $\prod_{v+1 \leqslant s \leqslant u-1} \sharp X_{u, s}^{k}=1$ if $v=u-1$.

If $p(u, v) \geqslant 2$, let

$$
N_{u, v}=N\left(p(u, v), 1+\max _{1 \leqslant j \leqslant p(u, v)} \prod_{k \in U(u, v, j)} \prod_{v+1 \leqslant s \leqslant u-1} \sharp X_{t, s}^{k}, t\right)
$$

let

$$
W(u, v, i)=\left(\prod_{k \in U(u, v, i)} \prod_{v+1 \leqslant s \leqslant u-1}^{\left[1, N_{u, v}\right]} \sharp X_{u, s}^{k}\right) \quad(1 \leqslant i \leqslant p(u, v))
$$

and let

$$
X(u, v, p(u, v), p(u, v)-1)=[1, \sharp W(u, v, 1) \cdot \sharp W(u, v, p(u, v))] .
$$

For $i \in[1, p(u, v)-1]$ let

$$
m(u, v, p(u, v), i))=H J(\sharp X(u, v, p(u, v), i), \sharp W(u, v, i))
$$

and let

$$
X(u, v, p(u, v), i-1)=(X(u, v, p(u, v), i))^{m(u, v, p(u, v), i)} .
$$

For $j \in[1, p(u, v)-1], i \in[1, j-1]$ let

$$
\begin{aligned}
X(u, v, j, j-1) & =[1, \sharp W(u, v, j) . \\
& \left.\prod_{j+1 \leqslant k \leqslant p(u, v)} \prod_{1 \leqslant h \leqslant j}\left\{(\sharp X(u, v, k, h)+1)^{m(u, v, k, h)}-(\sharp X(u, v, k, h))^{m(u, v, k, h)}\right\}\right]
\end{aligned}
$$

let

$$
m(u, v, j, i)=H J\left(\sharp X(u, v, j, i),(\sharp W(u, v, i))^{\prod_{j+1 \leqslant \beta \leqslant p(u, v)} \sharp X(u, v, h, i-1)}\right)
$$

and let

$$
X(u, v, j, i-1)=(X(u, v, j, i))^{m(u, v, j, i)}
$$

We set

$$
\begin{aligned}
& X(u, v, j)=X(u, v, j, 0) \\
& Y(u, v, j)=\left[1, N_{u, v}\right] \quad(1 \leqslant j \leqslant p(u, v)) .
\end{aligned}
$$


DEFINITION 3.2 .

$$
\begin{aligned}
& X^{k}=X^{k}\left(\tau_{1}, \ldots, \tau_{t}\right)=\prod_{u=2}^{t} \prod_{v=1}^{u-1} X_{u, v}^{k}\left(\tau_{1}, \ldots, \tau_{t}\right), \\
& Y^{k}=Y^{k}\left(\tau_{1}, \ldots, \tau_{t}\right)=\prod_{u=2}^{t} \prod_{v=1}^{u-1} Y_{u, v}^{k}\left(\tau_{1}, \ldots, \tau_{t}\right) \quad(1 \leqslant k \leqslant n) .
\end{aligned}
$$

\section{Let}

$$
\begin{aligned}
& \operatorname{pr}_{X(u, v, j)}: X^{\rho_{u, v}(j)} \longrightarrow X_{u, v}^{\rho_{u, v}(j)}, \\
& \operatorname{pr}_{Y(u, v, j)}: Y^{\rho_{u, v}(p(u, v)+j)} \longrightarrow Y_{u, v}^{\rho_{u, v}(p(u, v)+j)} \quad(1 \leqslant v<u \leqslant t, \quad 1 \leqslant j \leqslant p(u, v))
\end{aligned}
$$

be the projections.

Suppose that $\sharp Z^{k} \geqslant \sharp X^{k}+\sharp Y^{k}$ and let $\iota_{1}^{k}: X^{k} \longrightarrow Z^{k}, \iota_{2}^{k}: Y^{k} \longrightarrow Z^{k}$ be injections satisfying $\mathfrak{R}\left(\iota_{1}^{k}\right) \cap \mathfrak{R}\left(\iota_{2}^{k}\right)=\phi(1 \leqslant k \leqslant n)$. We identify $\iota_{1}^{k}\left(X^{k}\right)$ and $\iota_{2}^{k}\left(Y^{k}\right)$ with $X^{k}$ and $Y^{*}$ respectively.

In the following five definitions and two lemmas, $u, v \in[1, t]$ are taken so that $v<u, \quad p(u, v) \geqslant 2, \tau_{u} \notin \Sigma_{+}, \tau_{v} \in \Sigma_{+}$. Set

$$
\begin{aligned}
& \mathfrak{F}(u, v, k, j)=\bigcup_{J \subsetneq[1, m(u, v, k, j)]}{ }^{J} X(u, v, k, j) \quad(2 \leqslant k \leqslant p(u, v), 1 \leqslant j \leqslant k-1) \\
& \mathfrak{C}(f)=[1, m(u, v, k, j)] \backslash \mathcal{D}(f) \quad(f \in \mathfrak{F}(u, v, k, j)) .
\end{aligned}
$$

Definition 3.3. For $f_{1} \in \mathfrak{F}(u, v, k, 1), \ldots, f_{j} \in \mathfrak{F}(u, v, k, j) \quad(2 \leqslant k \leqslant p(u, v)$, $1 \leqslant j \leqslant k-1)$ we set

$$
\begin{aligned}
& L\left(f_{1}, \ldots, f_{j}\right)=\left\{x^{k} \in X(u, v, k) \mid\right. \\
& x_{\left(s_{1}, \ldots, s_{h}\right)}^{k}=f_{h}\left(s_{h}\right)\left(\left(s_{1}, \ldots, s_{h}\right) \in \mathfrak{C}\left(f_{1}\right) \times \cdots \times \mathfrak{C}\left(f_{h-1}\right) \times \mathfrak{D}\left(f_{h}\right)(1 \leqslant h \leqslant j)\right), \\
& \qquad \prod_{\left(s_{1}, \ldots, s_{j}\right) \in \mathbb{C}\left(f_{1}\right) \times \cdots \times \mathfrak{C}\left(f_{j}\right)}\left\{x_{\left(s_{1}, \ldots, s_{j}\right)}^{k} \in \bigcup_{w \in X(u, v, k, j)}\{w\}^{\left.\sharp \mathbb{C}\left(f_{1}\right) \cdots \cdots \mathbb{E}\left(f_{j}\right)\right\}}\right\} \\
& I\left(L\left(f_{1}, \ldots, f_{j}\right)\right)=\mathfrak{C}\left(f_{1}\right) \times \cdots \times \mathfrak{C}\left(f_{j}\right), \\
& J\left(L\left(f_{1}, \ldots, f_{j}\right)\right)=([1, m(u, v, k, 1)] \times \cdots \times[1, m(u, v, k, j)]) \backslash I\left(L\left(f_{1}, \ldots, f_{j}\right)\right)
\end{aligned}
$$




\section{DEFINITION 3.4. We set}

$$
\begin{aligned}
& \mathfrak{L}^{j}(X(u, v, k))=\left\{L\left(f_{1}, \ldots, f_{j}\right) \subset X(u, v, k) \mid f_{i} \in \mathfrak{F}(u, v, k, i) \quad(1 \leqslant i \leqslant j)\right\}, \\
& \mathfrak{L}^{j}(X(u, v, j+1) \times \cdots \times X(u, v, p(u, v))) \\
& =\left\{L_{j}^{j+1} \times \cdots \times L_{j}^{p(u, v)} \subset X(u, v, j+1) \times \cdots \times X(u, v, p(u, v)) \mid\right. \\
& \left.\quad L_{j}^{j+1} \in \mathfrak{L}^{j}(X(u, v, j+1)), \ldots, L_{j}^{p(u, v)} \in \mathfrak{L}^{j}(X(u, v, p(u, v)))\right\}
\end{aligned}
$$

and

$$
\begin{aligned}
& \mathfrak{L}(X(u, v, k, j-1))=\left\{\left\{\left(w_{1}, \ldots, w_{m(u, v, k, j}\right) \in X(u, v, k, j-1) \mid\right.\right. \\
&\left.w_{i}=f(i) \quad(i \in \mathfrak{D}(f)), \quad \prod_{i \in \mathfrak{C}(f)}\left\{w_{i}\right\} \in \bigcup_{w \in X(u, v, k, j)}\{w\}^{\sharp \mathbb{C}(f)}\right\} \\
&\subset X(u, v, k, j-1) \mid f \in \mathfrak{F}(u, v, k, j)\}(2 \leqslant k \leqslant p(u, v), 1 \leqslant j \leqslant k-1) .
\end{aligned}
$$

For $f_{1} \in \mathfrak{F}(u, v, k, 1), \ldots, f_{j} \in \mathfrak{F}(u, v, k, j)(2 \leqslant k \leqslant p(u, v), 1 \leqslant j \leqslant k-2)$ we set $\mathfrak{L}\left(L\left(f_{1}, \ldots, f_{j}\right)\right)=\left\{L\left(f_{1}, \ldots, f_{j}, f_{j+1}\right) \subset L\left(f_{1}, \ldots, f_{j}\right) \mid f_{j+1} \in \mathfrak{F}(u, v, k, j+1)\right\}$.

For $L_{j}^{i} \in \mathfrak{L}^{j}(X(u, v, i))(2 \leqslant i \leqslant p, 1 \leqslant j \leqslant i-1)$ let $\lambda_{L_{j}^{i}}$ be a bijection from $X(u, v, i, j)$ onto $L_{j}^{i}$ such that for all $w \in X(u, v, i, j)$

$$
x^{i}=\lambda_{L_{j}^{i}}(w) \Longleftrightarrow x_{\left(s_{i}, \ldots, s_{j}\right)}^{i}=w \quad\left(\left(s_{1}, \ldots, s_{j}\right) \in I\left(L_{j}^{i}\right)\right)
$$

Let

$$
\begin{gathered}
\alpha_{u, v}^{k}: \mathfrak{L}^{k}(X(u, v, k+1) \times \cdots \times X(u, v, p(u, v))) \times W(u, v, k) \\
\longrightarrow X(u, v, k, k-1) \quad(1 \leqslant k \leqslant p-1), \\
\alpha_{u, v}^{p}: W(u, v, 1) \times W(u, v, p(u, v)) \longrightarrow X(u, v, p(u, v), p(u, v)-1)
\end{gathered}
$$

be fixed bijections.

We make the following definitions depending on $\alpha_{u, v}^{1}, \alpha_{u, v}^{2}, \ldots, \alpha_{u, v}^{p(u, v)}$.

Definition 3.5. (1) Define mappings

$$
\begin{aligned}
Q^{u, v, k}: X(u, v, 1) & \cdots \times X(u, v, k) \\
\longrightarrow & \mathfrak{L}^{k}(X(u, v, k+1) \times \cdots \times X(u, v, p(u, v))) \cup\{\phi\} \\
& (1 \leqslant k \leqslant p(u, v)-1)
\end{aligned}
$$

by induction as follows.

For $a^{1} \in \alpha_{u, v}^{1}\left(\left\{L_{1}^{2} \times \cdots \times L_{1}^{p(u, v)}\right\} \times W(u, v, 1)\right)$ where $L_{1}^{j} \in \mathfrak{L}(X(u, v, j))$ $(2 \leqslant j \leqslant p(u, v))$,

$$
Q^{u, v, 1}\left(a^{1}\right)=L_{1}^{2} \times \cdots \times L_{1}^{p(u, v)} .
$$


For $k \in[2, p(u, v)-1]$ if

$$
\begin{gathered}
Q^{u, v, k-1}\left(a^{1}, \ldots, a^{k-1}\right)=L_{k-1}^{k} \times \cdots \times L_{k-1}^{p(u, v)} \\
\in \mathfrak{L}^{k-1}(X(u, v, k) \times \cdots \times X(u, v, p(u, v)))
\end{gathered}
$$

and

$$
a^{k} \in \lambda_{L_{k-1}^{k}} \circ \alpha_{u, v}^{k}\left(\left\{L_{k}^{k+1} \times \cdots \times L_{k}^{p(u, v)}\right\} \times W(u, v, k)\right)
$$

where $L_{k}^{j} \in \mathfrak{L}\left(L_{k-1}^{j}\right)(k+1 \leqslant j \leqslant p(u, v))$ then

$$
Q^{u, v, k}\left(a^{1}, \ldots, a^{k}\right)=L_{k}^{k+1} \times \cdots \times L_{k}^{p(u, v)} .
$$

Otherwise

$$
Q^{u, v, k}\left(a^{1}, \ldots, a^{k}\right)=\phi
$$

(2) Define a mapping

$$
Q^{u, v, p(u, v)}: X(u, v, 1) \times \cdots \times X(u, v, p(u, v)) \longrightarrow W(u, v, 1) \cup\{\phi\}
$$

as follows. If $a^{1}=\alpha_{u, v}^{1}\left(Q^{u, v, 1}\left(a^{1}\right), B\right)$ for some $B \in W(u, v, 1)$ and

$$
a^{p(u, v)} \in \lambda_{Q^{x \cdot v, p(x . v)-1}\left(\alpha^{1}, \ldots, a^{p(u, v)-1}\right)} \circ \alpha_{u, v}^{p(u, v)}(\{B\} \times W(u, v, p(u, v)))
$$

then

$$
Q^{u, v, p(u, v)}\left(a^{1}, \ldots, a^{p(u, v)}\right)=B
$$

Otherwise

$$
Q^{u, v, p(u, v)}\left(a^{1}, \ldots, a^{p(u, v)}\right)=\phi .
$$

(3) For $k \in[1, p(u, v)-1]$ and $\left(a^{1}, \ldots, a^{k}\right) \in X(u, v, 1) \times \cdots \times X(u, v, k)$ if $Q^{u, v, k}\left(a^{1}, \ldots, a^{k}\right)=L_{k}^{k+1} \times \cdots \times L_{k}^{p(u, v)} \in \mathfrak{L}^{k}(X(u, v, k+1) \times \cdots \times X(u, v, p(u, v)))$, let $Q_{1}^{u, v, k}\left(a^{1}, \ldots, a^{k}\right)=L_{k}^{k+1}$. If $Q^{u, v, k}\left(a^{1}, \ldots, a^{k}\right)=\phi$, let $Q_{1}^{u, v, k}\left(a^{1}, \ldots, a^{k}\right)=\phi$.

Definition 3.6. Define $\beta_{u, v}^{1}: X(u, v, 1) \longrightarrow W(u, v, 1)$ by $\beta_{u, v}^{1}\left(a^{1}\right)=B^{1}$ if $a^{1} \in$ $X(u, v, 1)$ and $B^{1} \in W(u, v, 1)$ are such that $a^{1}=\alpha_{u, v}^{1}\left(Q^{u, v, 1}\left(a^{1}\right), B^{1}\right)$.

For $k \in[2, p(u, v)]$ define

$$
\beta_{u, v}^{k}: X(u, v, 1) \times \cdots \times X(u, v, k) \longrightarrow W(u, v, k) \cup\{\phi\}
$$

by $\beta_{u, v}^{k}\left(a^{1}, \ldots, a^{k}\right)=B^{k}$ if $a^{1} \in X(u, v, 1), \ldots, a^{k} \in X(u, v, k)$ are such that

$$
a^{k}=\lambda_{Q_{1}^{u, v, k-1}\left(a^{1}, \ldots, a^{k-1}\right)} \circ \alpha_{u, v}^{k}\left(Q^{u, v, k}\left(a^{1}, \ldots, a^{k}\right), B^{k}\right),
$$

and $\beta_{u, v}^{k}\left(a^{1}, \ldots, a^{k}\right)=\phi$ otherwise.

\section{DEFINITION 3.7.}

$$
\begin{aligned}
T_{u, v} & =\left\{\left(x^{1}, \ldots, x^{p(u, v)}, y^{1}, \ldots, y^{p(u, v)}\right) \in X(u, v, 1) \times \cdots \times X(u, v, p(u, v))\right. \\
& \left.\times Y(u, v, 1) \times \cdots \times Y(u, v, p(u, v)) \mid y^{k} \in \beta_{u, v}^{k}\left(x^{1}, \ldots, x^{k}\right), 1 \leqslant k \leqslant p(u, v)\right\} .
\end{aligned}
$$


LEMMA 3.1. For each $k \in[1, p(u, v)]$ let $\varphi^{k}:\left[1, N_{u, v}\right]^{p(u, v)-1} \longrightarrow X(u, v, k)$ and let $S^{k} \subset\left[1, N_{u, v}\right]$ with $\sharp S^{k}>\prod_{h \in U(u, v, k)} \prod_{v+1 \leqslant s \leqslant u-1} \sharp X_{u, s}^{h}$. Then

$$
\begin{gathered}
\left\{\left(\varphi^{1}\left(y^{1}, y^{3}, \ldots, y^{p(u, v)}\right), \ldots, \varphi^{p(u, v)-1}\left(y^{1}, \ldots, y^{p(u, v)-1}\right), \varphi^{p(u, v)}\left(y^{2}, \ldots, y^{p(u, v)}\right)\right.\right. \\
\left.y^{1}, \ldots, y^{p(u, v)}\right) \in X(u, v, 1) \times \cdots \times X(u, v, p(u, v)) \times\left[1, N_{u, v}\right]^{p(u, v)} \\
\left.y^{k} \in S^{k}, 1 \leqslant k \leqslant p(u, v)\right\} \nsubseteq T_{u, v} .
\end{gathered}
$$

Proof. To obtain a contradiction assume that

$$
\begin{array}{r}
\left\{\left(\varphi^{1}\left(y^{1}, y^{3}, \ldots, y^{p(u, v)}\right), \ldots, \varphi^{p(u, v)-1}\left(y^{1}, \ldots, y^{p(u, v)-1}\right), \varphi^{p(u, v)}\left(y^{2}, \ldots, y^{p(u, v)}\right),\right.\right. \\
\left.y^{1}, \ldots, y^{p(u, v)}\right) \in X(u, v, 1) \times \cdots \times X(u, v, p(u, v)) \times\left[1, N_{u, v}\right]^{p(u, v)} \\
\left.y^{k} \in S^{k}, 1 \leqslant k \leqslant p(u, v)\right\} \subset T_{u, v} .
\end{array}
$$

For $k \in[1, p(u, v)]$ let

$$
\begin{aligned}
& B^{k}\left(y^{1}, \ldots, y^{p(u, v)}\right) \\
& =\beta_{u, v}^{k}\left(\varphi^{1}\left(y^{1}, y^{3}, \ldots, y^{p(u, v)}\right), \ldots, \varphi^{k}\left(y^{1}, \ldots, y^{k}, y^{k+2}, \ldots, y^{p(u, v)}\right)\right) \\
& \quad\left(y^{j} \in S^{j}, 1 \leqslant j \leqslant p(u, v)\right) .
\end{aligned}
$$

Then

$$
\sharp B^{k}\left(y^{1}, \ldots, y^{p(u, v)}\right)=\prod_{h \in U(u, v, k)} \prod_{v+1 \leqslant s \leqslant u-1} \sharp X_{u, s}^{h}<\sharp S^{k}
$$

and since the assumption implies that

$$
y^{k} \in B^{k}\left(y^{1}, \ldots, y^{p(u, v)}\right) \quad\left(y^{j} \in S^{j}, 1 \leqslant j \leqslant p(u, v)\right)
$$

we see that for all $y^{1} \in S^{1}, \ldots, y^{k-1} \in S^{k-1}, y^{k+1} \in S^{k+1}, \ldots, y^{p(u, v)} \in S^{p(u, v)}$ there exist $d_{1}^{k}, d_{2}^{k} \in S^{k}$ such that

$$
B^{k}\left(y^{1}, \ldots, y^{k-1}, d_{1}^{k}, y^{k+1}, \ldots, y^{p(u, v)}\right) \neq B^{k}\left(y^{1}, \ldots, y^{k-1}, d_{2}^{k}, y^{k+1}, \ldots, y^{p(u, v)}\right) .
$$

Then in a similar way as in the proof of Lemma 4.3 in Maruyama (2000) we have

$$
\beta_{u, v}^{\mathbf{l}}\left(\varphi^{1}\left(d_{1}^{\mathbf{l}}, y^{3}, \ldots, y^{p(u, v)}\right)\right)=\beta_{u, v}^{1}\left(\varphi^{1}\left(d_{2}^{1}, y^{3}, \ldots, y^{p(u, v)}\right)\right)
$$

for all $d_{1}^{1}, d_{2}^{1} \in S_{1}$, a contradiction to the above result for $k=1$.

LeMma 3.2. For all

$$
\begin{array}{r}
\varphi^{k}: X(u, v, 1) \times \cdots \times X(u, v, k-1) \times X(u, v, k+1) \times \cdots \times X(u, v, p(u, v)) \\
\longrightarrow W(u, v, k) \\
(1 \leqslant k \leqslant p)
\end{array}
$$

there exist $a^{1} \in X(u, v, 1), \ldots, a^{p(u, v)} \in X(u, v, p(u, v))$ such that

$$
\left(a^{1}, \ldots, a^{p(u, v)}, \varphi^{1}\left(a^{2}, \ldots, a^{p(u, v)}\right), \ldots, \varphi^{p(u, v)}\left(a^{1}, \ldots, a^{p(u, v)-1}\right)\right) \subset T_{u, v} .
$$


Proof. By using the theorem of Hales-Jewett, the proof is given similary as in the proof of Lemma 4.4 in Maruyama (2000) and is omitted.

Definition 3.8. For $u, v \in[1, t]$ with $v<u$ define $\mathfrak{T}_{u, v} \subset Z^{1} \times \cdots \times Z^{n}$ as follows. If $p(u, v)=0$, then

$$
\mathfrak{T}_{u, v}=\left\{\left(z^{1}, \ldots, z^{n}\right) \in Z^{1} \times \cdots \times Z^{n} \mid z^{\rho_{u . v}} \notin Y^{\rho_{u, v}}\right\} .
$$

If $p(u, v) \geqslant 1, \tau_{u} \in \Sigma_{+}$, then

$$
\begin{aligned}
\mathcal{T}_{u, v}=\{ & \left(z^{1}, \ldots, z^{n}\right) \in Z^{1} \times \cdots \times Z^{n} \mid \\
& z^{\rho_{u, v}(k)} \in X^{\rho_{u, v}}, z^{\rho_{u, v}(p(u, v)+k)} \in Y^{\rho_{u, v}(p(u, v)+k)}, z_{u, v}^{\rho_{u, v}(k)}=z_{u, v}^{\rho_{u, v}(p(u, v)+k),} \\
1 \leqslant k \leqslant p(u, v)\} & 1 \leqslant Z^{n} \mid \\
\cup & \left(z^{1}, \ldots, z^{n}\right) \in Z^{1} \times \cdots \times Z^{n} \\
& \left.\left(z^{\rho_{u, v}(p(u, v)+1)}, \ldots, z^{\rho_{u, v}(2 p(u, v))}\right) \notin Y^{\rho_{u, v}(p(u, v)+1)} \times \cdots \times Y^{\rho_{u, v}(2 p(u, v)\}}\right\} .
\end{aligned}
$$

If $p(u, v)=1, \tau_{u} \notin \Sigma_{+}$, then

$$
\begin{aligned}
& \mathfrak{T}_{u, v}=\left\{\left(z^{1}, \ldots, z^{n}\right) \in Z^{1} \times \cdots \times Z^{n} \mid\right. \\
&\left.z^{\rho_{u, v}(1)} \in X^{\rho_{u, v}(1)}, z^{\rho_{u, v}(2)} \in Y^{\rho_{u, v}(2)}, z_{u, v}^{\rho_{u, v}(2)} \in \beta_{u, v}^{1}\left(z_{u, v}^{\rho_{u, v}(1)}\right)\right\} \\
& \cup\left\{\left(z^{1}, \ldots, z^{n}\right) \in Z^{1} \times \cdots \times Z^{n} \mid z^{\rho_{u, v}(2)} \notin Y^{\rho_{u, v}(2)}\right\}
\end{aligned}
$$

where $\beta_{u, v}^{1}: X(u, v, 1) \longrightarrow W(u, v, 1)$ is a fixed bijection.

If $p(u, v) \geqslant 2, \tau_{u} \notin \Sigma_{+}$then define $\mathfrak{T}_{u, v}$ by

$$
\begin{aligned}
& \mathfrak{T}_{u, v}=\left\{\left(z^{1}, \ldots, z^{n}\right) \in Z^{1} \times \cdots \times Z^{n} \mid\right. \\
& z^{\rho_{u, v}(k)} \in X^{\rho_{u, v}(k)}, z^{\rho_{u, v}(p(u, v)+k)} \in Y^{\rho_{u, v}(p(u, v)+k)}, \quad 1 \leqslant k \leqslant p(u, v), \\
&\left.\quad\left(z_{u, v}^{\rho_{u, v}(1)}, \ldots, z_{u, v}^{\rho_{u, v}(2 p(u, v))}\right) \in T_{u, v}\right\} \\
& \cup\left\{\left(z^{1}, \ldots, z^{n}\right) \in Z^{1} \times \cdots \times Z^{n} \mid\right. \\
&\left.\quad\left(z^{\rho_{u, v}(p(u, v)+1)}, \ldots, z^{\rho_{u, v}(2 p(u, v))}\right) \notin Y^{\rho_{u, v}(p(u, v)+1)} \times \cdots \times Y^{\rho_{u, v}(2 p(u, v))}\right\} .
\end{aligned}
$$

For $1 \leqslant v<u \leqslant t$ define functions $F_{0}^{u, v}, F_{0}^{u}, F_{0}: Z^{1} \times \cdots \times Z^{n} \rightarrow R$ by

$$
\begin{aligned}
& F_{0}^{u, v}\left(z^{1}, \ldots, z^{n}\right)= \begin{cases}1 & \left(\left(z^{1}, \ldots, z^{n}\right) \in \mathfrak{T}_{u, v}\right), \\
0 & \left(\left(z^{1}, \ldots, z^{n}\right) \notin \mathfrak{T}_{u, v}\right),\end{cases} \\
& F_{0}^{u}\left(z^{1}, \ldots, z^{n}\right)=\min _{1 \leqslant v \leqslant u-1} F_{0}^{u, v}\left(z^{1}, \ldots, z^{n}\right), \\
& F_{0}\left(z^{1}, \ldots, z^{n}\right)=\max _{2 \leqslant u \leqslant t}(u-1) F_{0}^{u}\left(z^{1}, \ldots, z^{n}\right) .
\end{aligned}
$$


LEMMA 3.3. Let $1 \leqslant s<u \leqslant t$ and $\tau_{s}=\sigma_{+}\left(l_{s}, \pi_{s}, \gamma^{s}\right)$. Then for all $\varphi^{1} \in$ $\Phi\left(\pi_{s}(1), \gamma_{1}^{s}\right), \varphi^{2} \in \Phi\left(\pi_{s}(2), \gamma_{2}^{s}\right), \ldots, \varphi^{l_{s}} \in \Phi\left(\pi_{s}\left(l_{s}\right), \gamma_{i_{s}}^{s}\right)$,

$$
\begin{gathered}
\sharp\left\{\left(z^{\pi_{s}\left(l_{s}+1\right)}, \ldots, z^{\pi_{s}(n)}\right) \in Y^{\pi_{*}\left(l_{s}+1\right)} \times \cdots \times Y^{\pi_{A}(n)} \mid\right. \\
\left.\quad\left(\gamma^{s} * \pi_{s} * F_{0}^{u, s}\right)\left(\varphi^{1}, \ldots, \varphi^{l_{s}}, z^{\pi_{s}\left(l_{s}+1\right)}, \ldots, z^{\pi_{s}(n)}\right)=1\right\} \\
\leqslant \frac{1}{t} \cdot \sharp Y^{\pi_{s}\left(l_{s}+1\right)} \cdots \cdots \sharp Y^{\pi_{s}(n)} .
\end{gathered}
$$

Proof. Suppose that $p(u, s) \geqslant 2$. Let $\varphi^{j} \in \Phi\left(\pi_{s}(j), \gamma_{j}^{s}\right)\left(1 \leqslant j \leqslant l_{s}\right)$ let

$$
\begin{aligned}
& S^{i}=\{1, \ldots, i, i+2, \ldots, p(u, s)\} \quad(1 \leqslant i \leqslant p(u, s)-1), \\
& S^{p(u, s)}=\{2, \ldots, p(u, s)\}
\end{aligned}
$$

and let

$$
\boldsymbol{b}=\left(b_{v, r}^{k}\right)_{(k, v, r) \in V(s, u)} \in \prod_{\{k, v, r) \in V(s, u)} Y_{v, r}^{k} .
$$

We may assume that $\mathfrak{R}\left(\varphi^{j}\right) \subset X^{\pi_{s}(j)}\left(1 \leqslant j \leqslant l_{s}\right)$. Define

$$
\varphi_{b}^{\pi_{s}^{-1} \circ \rho_{u . .(i)}}: \prod_{j \in S^{i}} Y(u, s, j) \longrightarrow X^{\rho_{u . s}(i)}(1 \leqslant i \leqslant p(u, s))
$$

by

$$
\varphi_{b}^{\pi^{-1} \propto \rho_{u, s}(i)}\left(\left(y^{j}\right)_{j \in S^{i}}\right)=\varphi^{\pi_{0}^{-1} \circ \rho_{u . s}(i)}\left(\left(z^{k}\right)_{k \in U(s, u, i)}\right)
$$

where $\left(z^{k}\right)_{k \in U(s, u, i)} \in \prod_{k \in U(s, u, i)} Y^{k}$ is such that

$$
z_{v, r}^{k}=b_{v, r}^{k} \quad(k \in U(s, u, i),(k, v, r) \in V(s, u)), \quad z_{u, s}^{\rho_{u, a}(j)}=y^{j} \quad\left(j \in S^{i}\right) .
$$

The definition of $N_{u, s}$ and Lemma 3.1 imply that

$$
\begin{aligned}
& \sharp\left\{\left(y^{1}, \ldots, y^{p(u, s)}\right) \in Y(u, s, 1) \times \cdots \times Y(u, s, p(u, s)) \mid\right. \\
& \left(\operatorname{pr}_{X(u, s, 1)} \circ \varphi_{b}^{\pi_{b}^{-1} \circ \rho_{u . s}(1)}\left(y^{1}, y^{3}, \ldots, y^{p(u, s)}\right), \ldots,\right. \\
& \left.\left.\operatorname{pr}_{X(u, s, p(u, s))} \circ \varphi_{b}^{\pi_{s}^{-1} \circ \rho_{u . s}(p(u, s))}\left(y^{2}, \ldots, y^{p(u, s)}\right), y^{1}, \ldots, y^{p(u, s)}\right) \in T_{u, s}\right\} \\
& \leqslant \frac{1}{t} \cdot \sharp Y(u, s, 1) \cdots \cdots \sharp Y(u, s, p(u, s)) .
\end{aligned}
$$

Hence

$$
\begin{aligned}
& \sharp\left\{\left(z^{\pi_{s}\left(l_{s}+1\right)}, \ldots, z^{\pi_{s}(n)}\right) \in Y^{\pi_{s}\left(l_{s}+1\right)} \times \cdots \times Y^{\pi_{s}(n)} \mid\right. \\
& \left.\left(\gamma^{s} * \pi_{s} * F_{0}^{u, s}\right)\left(\varphi^{1}, \ldots, \varphi^{l_{s}}, z^{\pi_{s}\left(l_{s}+1\right)}, \ldots, z^{\pi_{s}(n)}\right)=1\right\} \\
& \leqslant \frac{1}{t} \cdot \sharp Y^{\pi_{s}\left(l_{s}+1\right)} \cdots \cdots \sharp Y^{\pi_{s}(n)} .
\end{aligned}
$$

The lemma is clear when $p(u, s) \leqslant 1$. 
Lemma 3.4. For $s \in[1, t]$ if $\tau_{s}=\sigma_{-}\left(l_{s}, \pi_{s}, \gamma^{s}\right) \in \Sigma \backslash \Sigma_{+}$then for all $\varphi^{1} \in$ $\Phi\left(\pi_{s}(1), \gamma_{1}^{s}\right), \ldots, \varphi^{l_{*}} \in \Phi\left(\pi_{s}\left(l_{s}\right), \gamma_{l}^{s}\right)$ there exist $a^{\pi_{*}\left(l_{*}+1\right)} \in X^{\pi_{s}\left(l_{s}+1\right)}, \ldots, a^{\pi_{*}(n)} \in$ $X^{\pi_{A}(n)}$ such that

$$
\left(\gamma^{s} * \pi_{s} * F_{0}^{s}\right)\left(\varphi^{1}, \ldots, \varphi^{l *}, a^{\pi_{s}\left(l_{s}+1\right)}, \ldots, a^{\pi_{s}(n)}\right)=1 .
$$

Proof. Let $\varphi^{h} \in \Phi\left(\pi_{s}(h), \gamma_{h}^{s}\right)\left(1 \leqslant h \leqslant l_{s}\right)$. We may suppose that $\mathfrak{R}\left(\varphi^{h}\right) \subset$ $Y^{\pi_{s}(h)}\left(1 \leqslant h \leqslant l_{s}\right)$.

We claim that for each $v \in[0, s-1]$ there exists

$$
a(v)=\left(a_{u, r}^{k}\right)_{(k, u, r) \in V(s, v)} \in \prod_{(k, u, r) \in V(s, v)} X_{u, r}^{k}
$$

such that for all

$$
\left(z^{\pi_{s}\left(l_{s}+1\right)}, \ldots, z^{\pi_{\theta}(n)}\right) \in Z^{\pi_{s}\left(l_{\theta}+1\right)} \times \cdots \times Z^{\pi_{s}(n)}
$$

if

$$
\left(z_{u, r}^{k}\right)_{(k, u, r) \in V(s, v)}=a(v)
$$

then

$$
\left(\gamma^{s} * \pi_{s} * F_{0}^{s, q}\right)\left(\varphi^{1}, \ldots, \varphi^{l_{s}}, z^{\pi_{s}\left(l_{s}+1\right)}, \ldots, z^{\pi_{s}(n)}\right)=1
$$

for all $q=1,2, \ldots, v$.

We proceed by induction on $v$. The case $v=0$ is trivially true for every $a(0) \epsilon$ $\prod_{(k, u, r) \in V(s, 0)} X_{u, r}^{k}$. We assume this is true for $v-1$ and prove this for $v$. The case $p(s, v)=0$ is trivial. We suppose that $p(s, v) \geqslant 1$. Define

$$
\varphi_{a(v-1)}^{\pi_{s}^{-1} \circ \rho_{s, v}(p(s, v)+j)}: \prod_{k \in U(s, v, j)} \prod_{v \leqslant r \leqslant s-1} X_{s, r}^{k} \longrightarrow Y^{\rho_{s, v}(p(s, v)+j)} \quad(1 \leqslant j \leqslant p(s, v))
$$

by

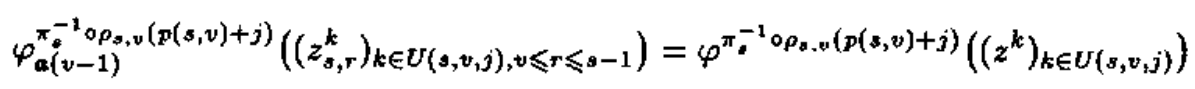

where $\left(z^{k}\right)_{k \in U(s, v, j)} \in \prod_{k \in U(s, v, j)} X^{k}$ is such that

$$
z_{u, r}^{k}=a_{u, r}^{k} \quad(k \in U(s, v, j),(k, u, r) \in V(s, v-1)) .
$$

If $p(s, v)=1$, then since

$$
\sharp\left(\prod_{k \in U(s, v, 1)} \prod_{v \leqslant r \leqslant s-1} X_{s, r}^{k}\right)=\prod_{k \in U(s, v, 1)} \prod_{v+1 \leqslant r \leqslant s-1} \sharp X_{s, r}^{k},
$$

we can take $a_{s, v}^{k} \in X_{s, v}^{k}\left(k \in \pi_{s}\left(\left[l_{s}+1, n\right]\right)\right)$ so that

$$
\operatorname{pr}_{Y(s, v, 1)} \circ \varphi_{a(v-1)}^{\pi_{s}^{-1} \circ \rho_{s, v}(2)}\left(\prod_{k \in U(s, v, 1)} \prod_{v \leqslant r \leqslant s-1} X_{s, r}^{k}\right) \subset \beta_{s, v}^{1}\left(a_{s, v}^{\rho(1)}\right) .
$$


Let $a(v)=\left(a_{u, r}^{k}\right)_{(k, u, r) \in V(s, v)}$ then if

$$
\left(z^{\pi_{*}\left(l_{A}+1\right)}, \ldots, z^{\pi_{s}(n)}\right) \in Z^{\pi_{*}\left(l_{s}+1\right)} \times \cdots \times Z^{\pi_{s}(n)}
$$

is such that

$$
\left(z_{u, r}^{k}\right)_{(k, u, r) \in V(s, v)}=\mathbf{a}(v)
$$

then

$$
\left(\gamma^{s} * \pi_{s} * F_{0}^{s, q}\right)\left(\varphi^{1}, \ldots, \varphi^{l_{s}}, z^{\pi_{*}\left(l_{*}+1\right)}, \ldots, z^{\pi_{s}(n)}\right)=1
$$

for all $q=1,2, \ldots, v$.

If $p(s, v) \geqslant 2$, let

$$
\begin{aligned}
\Psi^{j}: X(s, v, 1) \times \cdots \times X(s, v, j-1) \times X(s, v, j+1) \times \cdots \times X(s, v, p(s, v)) & \\
& \longrightarrow W(s, v, j)
\end{aligned}
$$

be such that for all $x^{1} \in X(s, v, 1), x^{2} \in X(s, v, 2), \ldots, x^{p(s, v)} \in X(s, v, p(s, v))$

$$
\begin{aligned}
& \operatorname{pr}_{Y(s, v, j)} \circ \varphi_{a(v-1)}^{\pi_{s}^{-1} \circ \rho_{v, v}(p(s, v)+j)} \\
& \left(\left\{\left(z_{s, r}^{k}\right)_{k \in U(s, v, j), v \leqslant r \leqslant s-1} \in \prod_{k \in U(s, v, j)} \prod_{v \leqslant r \leqslant s-1} X_{s, r}^{k} \mid z_{s, v}^{\rho_{s, v}(i)}=x^{i} \quad(1 \leqslant i \leqslant p(s, v))\right\}\right) \\
& \quad \subset \Psi^{j}\left(x^{1}, \ldots, x^{j-1}, x^{j+1}, \ldots, x^{p(s, v)}\right) \quad(1 \leqslant j \leqslant p(s, v)) .
\end{aligned}
$$

By Lemma 3.2 there exist $e^{1} \in X(s, v, 1), \ldots, e^{p(s, v)} \in X(s, v, p(s, v))$ such that

$$
\left(e^{1}, \ldots, e^{p(s, v)}, \Psi^{1}\left(e^{2}, \ldots, e^{p(s, v)}\right), \ldots, \Psi^{p(s, v)}\left(e^{1}, \ldots, e^{p(s, v)-1}\right)\right) \subset T_{s, v} .
$$

Take

$$
a_{s, v}^{k} \in X_{s, v}^{k}\left(k \in \pi_{s}\left(\left[l_{s}+1, n\right]\right)\right)
$$

so that

$$
a_{s, v}^{\rho_{s, v}(j)}=e^{j}(1 \leqslant j \leqslant p(s, v))
$$

and let

$$
\boldsymbol{a}(v)=\left(a_{u, r}^{k}\right)_{(k, u, r) \in V(s, v)} .
$$

Then if

$$
\left(z^{\pi_{s}\left(l_{\theta}+1\right)}, \ldots, z^{\pi_{e}(n)}\right) \in Z^{\pi_{\theta}\left(l_{\bullet}+1\right)} \times \cdots \times Z^{\pi_{\theta}(n)}
$$

is such that

$$
\left(z_{u, r}^{k}\right)_{(k, u, r) \in V(s, v)}=a(v)
$$


then

$$
\left(\gamma^{s} * \pi_{s} * F_{0}^{s, q}\right)\left(\varphi^{1}, \ldots, \varphi^{l_{\theta}}, z^{\pi_{*}\left(l_{*}+1\right)}, \ldots, z^{\pi_{*}(n)}\right)=1
$$

for all $q=1,2, \ldots, v$, completing the induction.

By the claim there exists

$$
a(s-1)=\left(a^{k}\right)_{k \in \pi_{s}\left(\left[l_{s}+1, n\right]\right)} \in \prod_{k \in \pi_{s}\left(\left[l_{s}+1, n\right]\right)} X^{k}
$$

such that

$$
\left(\gamma^{s} * \pi_{s} * F_{0}^{s, q}\right)\left(\varphi^{1}, \ldots, \varphi^{l_{s}}, a^{\pi_{s}\left(l_{s}+1\right)}, \ldots, a^{\pi_{s}(n)}\right)=1
$$

for all $q=1,2, \ldots, v$ and since $F_{0}^{s}=\min _{1 \leqslant q \leqslant s-1} F_{0}^{s, q}$, we have

$$
\left(\gamma^{s} * \pi_{s} * F_{0}^{s}\right)\left(\varphi^{1}, \ldots, \varphi^{t_{s}}, a^{\pi_{s}\left(l_{s}+1\right)}, \ldots, a^{\pi_{s}(n)}\right)=1,
$$

completing the proof.

\section{Proof of the Ordering Theorem}

Let

$$
\begin{aligned}
K=\max \left\{\sharp X^{k}\left(\tau_{1}, \ldots, \tau_{t}\right)+\sharp Y^{k}\left(\tau_{1}, \ldots, \tau_{t}\right) \in N \mid\right. \\
\left.\quad \tau_{1}, \ldots, \tau_{t} \in \Sigma, \tau_{j} \leqslant \tau_{i}(1 \leqslant i<j \leqslant t), 1 \leqslant t \leqslant \sharp \Sigma, 1 \leqslant k \leqslant n\right\}
\end{aligned}
$$

and let $\sharp Z^{1} \geqslant K, \ldots, \sharp Z^{n} \geqslant K$. Let $\tau_{1}, \ldots, \tau_{t} \in \Sigma$ be given with $\tau_{j} \notin \tau_{i}(1 \leqslant i<j \leqslant t)$ and let $F_{0}$ be defined as before. We will prove $\tau_{s}\left(F_{0}\right)=s-1(1 \leqslant s \leqslant t)$. There are two cases.

Case 1. $\tau_{s}=\sigma_{-}\left(l_{s}, \pi_{s}, \gamma^{s}\right) \in \Sigma \backslash \Sigma+$ Let

$$
\varphi_{0}^{j}: \prod_{k \in \mathfrak{M}\left(\gamma_{j}^{j}\right)} Z^{k} \longrightarrow Y^{\pi_{s}(j)} \quad\left(1 \leqslant j \leqslant l_{s}\right)
$$

be such that, if $\tau_{u} \in \Sigma_{+}, p(u, s) \geqslant 1$, then

$$
\begin{gathered}
\operatorname{pr}_{Y(u, s, j)} \circ \varphi_{0}^{\pi_{s}^{-1} \circ \rho_{v, s}(p(u, s)+j)}\left(\left(z^{k}\right)_{k \in U(s, v, j)}\right)=z_{u, s}^{\rho_{u, s}(j-1)} \\
\left(z^{\rho_{u . s}(j-1)} \in X^{\rho_{u, s}(j-1)}, 2 \leqslant j \leqslant p(u, s)\right), \\
\operatorname{pr}_{Y(u, s, 1)} \circ \varphi_{0}^{\pi_{s}^{-1} \circ \rho_{u, s}(p(u, s)+1)}\left(\left(z^{k}\right)_{k \in U(s, u, 1)}\right) \neq z_{u, s}^{\rho_{u, s}(p(u, s))} \\
\left(z^{\rho_{u, s}(p(u, s))} \in X^{\rho_{u, s}(p(u, s))}\right),
\end{gathered}
$$

and if $\tau_{u} \notin \Sigma_{+}, p(u, s)=1$, then

$$
\operatorname{pr}_{Y(u, s, 1)} \circ \varphi_{0}^{\pi^{-1} \circ \rho_{u, g}(2)}\left(\left(z^{k}\right)_{k \in U(s, u, 1)}\right) \notin \beta_{u, s}^{1}\left(z_{u, s}^{\rho_{u, k}(1)}\right) \quad\left(z^{\rho_{u . t}(1)} \in X^{\rho_{u . s}(1)}\right) \text {. }
$$


Then it is clear that for all $z^{\pi_{s}\left(l_{A}+1\right)} \in Z^{\pi_{s}\left(l_{s}+1\right)}, z^{\pi_{*}\left(l_{s}+2\right)} \in Z^{\pi_{s}\left(l_{s}+2\right)}, \ldots, z^{\pi_{s}(n)} \in$ $Z^{\pi_{*}(n)}$ and for all $u=s+1, s+2, \ldots, t$

$$
\left(\gamma^{s} * \pi_{s} * F_{0}^{u, s}\right)\left(\varphi_{0}^{1}, \ldots, \varphi_{0}^{l_{s}}, z^{\pi_{s}\left(l_{s}+1\right)}, \ldots, z^{\pi_{A}(n)}\right)=0
$$

Thus $\tau_{s}\left(F_{0}\right) \leqslant s-1$. Lemma 3.4 gives $\tau_{s}\left(F_{0}\right) \geqslant s-1$.

Case 2. $\tau_{s}=\sigma_{+}\left(l_{s}, \pi_{s}, \gamma^{s}\right) \in \Sigma_{+}$. Let

$$
\varphi_{0}^{j}: \prod_{k \in \mathfrak{R}\left(\gamma_{j}^{*}\right)} Z^{k} \longrightarrow X^{\pi_{A}(j)} \quad\left(1 \leqslant j \leqslant l_{s}\right)
$$

be such that if $p(s, v) \geqslant 1$, then

$$
\begin{aligned}
\operatorname{pr}_{X(s, v, j)} \circ \varphi_{0}^{\pi_{s}^{-1} \circ \rho_{s . v}(j)}\left(\left(z^{k}\right)_{k \in U(s, v, j)}\right)=z_{s, v}^{\rho_{s, v}(p(s, v)+j)} \\
\left(z^{\rho_{s, v}(p(s, v)+j)} \in Y^{\rho_{\Delta, v}(p(s, v)+j)}, 1 \leqslant j \leqslant p(s, v)\right) .
\end{aligned}
$$

Then it is clear that for all $z^{\pi_{s}\left(l_{s}+1\right)} \in Z^{\pi_{s}\left(l_{s}+1\right)}, z^{\pi_{0}\left(l_{s}+2\right)} \in Z^{\pi_{s}\left(l_{s}+2\right)}, \ldots, z^{\pi_{s}(n)} \in$ $Z^{\pi_{s}(n)}$ and for all $v=1,2, \ldots, s-1$

$$
\left(\gamma^{s} * \pi_{s} * F_{0}^{s, v}\right)\left(\varphi_{0}^{1}, \ldots, \varphi_{0}^{l_{s}}, z^{\pi_{s}\left(l_{s}+1\right)}, \ldots, z^{\pi_{s}(n)}\right)=1
$$

Thus $\tau_{s}\left(F_{0}\right) \geqslant s-1$.

For $\varphi^{1} \in \Phi\left(\pi_{s}(1), \gamma_{1}^{s}\right), \ldots, \varphi^{l_{s}} \in \Phi\left(\pi_{s}\left(l_{s}\right), \gamma_{l_{s}}^{s}\right)$, by using Lemma 3.3,

$$
\begin{aligned}
& \sharp\left\{\left(z^{\pi_{s}\left(l_{s}+1\right)}, \ldots, z^{\pi_{s}(n)}\right) \in Y^{\pi_{s}\left(l_{s}+1\right)} \times \cdots \times Y^{\pi_{s}(n)} \mid\right. \\
& \left.\quad\left(\gamma^{s} * \pi_{s} * F_{0}\right)\left(\varphi^{1}, \ldots, \varphi^{l_{s}}, z^{\pi_{s}\left(l_{s}+1\right)}, \ldots, z^{\pi_{s}(n)}\right)>s-1\right\} \\
& \leqslant \sum_{u=s+1}^{t} \sharp\left\{\left(z^{\pi_{s}\left(l_{s}+1\right)}, \ldots, z^{\pi_{s}(n)}\right) \in Y^{\pi_{s}\left(l_{s}+1\right)} \times \cdots \times Y^{\pi_{s}(n)} \mid\right. \\
& \left.\quad\left(\gamma^{s} * \pi_{s} * F_{0}^{u, s}\right)\left(\varphi^{1}, \ldots, \varphi^{l_{s}}, z^{\pi_{s}\left(l_{s}+1\right)}, \ldots, z^{\pi_{s}(n)}\right)=1\right\} \\
& \leqslant \frac{t-s}{t} \cdot \sharp Y^{\pi_{s}\left(l_{s}+1\right)} \ldots \ldots \sharp Y^{\pi_{s}(n)} \\
& <\sharp Y^{\pi_{s}\left(l_{s}+1\right)} \ldots \ldots \sharp Y^{\pi_{s}(n)} .
\end{aligned}
$$

Hence $\tau_{s}\left(F_{0}\right) \leqslant s-1$, completing the proof.

\section{References}

Graham, R. L., Rothschild, B. L. and Spencer, J. H. (1990), Ramsey Theory, Second Edition. John Wiley \& Sons, New York.

Hisano, H. and Maruyama, F. (1989), On the order of max-min functionals. Bulletin of Informatics and Cybernetics, 23, 163-169.

Krynicki, M. and Mostowski, M. (1995), Henkin quantifiers. in: M. Krynicki, M.

Mostowski and L. W. Szczerba, eds., Quantifiers: Logics, Models and 
Computation. (Kluwer Academic Publishers, Dordrecht) 193-262.

Maruyama, F. (2000), An application of a combinatorial theorem to a separation problem: I. Bulletin of Informatics and Cybernetics, 32, 61-79.

Reveived December 22, 1999

Revised November 9, 2000

Re-revised March 1, 2001 